\title{
Advancements in nano-enabled therapeutics for neuroHIV management
}

\author{
This article was published in the following Dove Press journal: \\ International Journal of Nanomedicine \\ I September 2016 \\ Number of times this article has been viewed
}

\author{
Ajeet Kaushik \\ Rahul Dev Jayant \\ Madhavan Nair \\ Center for Personalized \\ NanoMedicine, Institute of \\ Neurolmmune Pharmacology, \\ Department of Immunology, Herbert \\ Wertheim College of Medicine, \\ Florida International University, \\ Miami, FL, USA
}

\begin{abstract}
This viewpoint is a global call to promote fundamental and applied research aiming toward designing smart nanocarriers of desired properties, novel noninvasive strategies to open the blood-brain barrier (BBB), delivery/release of single/multiple therapeutic agents across the BBB to eradicate neurohuman immunodeficiency virus (HIV), strategies for on-demand site-specific release of antiretroviral therapy, developing novel nanoformulations capable to recognize and eradicate latently infected HIV reservoirs, and developing novel smart analytical diagnostic tools to detect and monitor HIV infection. Thus, investigation of novel nanoformulations, methodologies for site-specific delivery/release, analytical methods, and diagnostic tools would be of high significance to eradicate and monitor neuroacquired immunodeficiency syndrome. Overall, these developments will certainly help to develop personalized nanomedicines to cure HIV and to develop smart HIV-monitoring analytical systems for disease management.
\end{abstract}

Keywords: nanotherapeutics, personalized nanomedicine, smart monitoring analytical systems, HIV disease management

\section{Discussion}

Human immunodeficiency virus (HIV) is a deadly infectious disease and has been declared as a serious concern worldwide. ${ }^{1-3}$ The World Health Organization confirms $>35$ million HIV-infected people. Among them, 28 million people are eligible for antiretroviral therapy (ART). However, only 11.7 million could afford antiretroviral (ARV) drugs. In spite of significant advances in highly active ART, the elimination of HIV-1 reservoirs from the peripheral nervous system and central nervous system (CNS) remains an ultimate challenge. ${ }^{4}$ This is attributed to the integration of HIV-1 genome with host genome, thereby causing viral latency in the periphery and in brain, as well. Furthermore, the inability of ART to penetrate the blood-brain barrier (BBB) after systemic administration makes brain as one of the most dominant HIV infection reservoirs. ${ }^{1}$ Developing the best suitable therapeutics for HIV demands multidisciplinary research and development approaches involving smart sensors, novel imaging agents, smart portable diagnostic tools, biomarkers, novel bioimaging, efficient affinity agents, smart assaying, real-time disease monitoring, and disease management systems to develop accessible cost-effective diagnosis and treatment for HIV patients. ${ }^{1}$

Advancements in analysis have proven a significant role in HIV diagnostics and monitoring of disease progression. The US Food and Drug Administration approved $\sim 38$ various anti-HIV drugs. The therapeutic effects of these drugs have been studied using advanced analytical tools and methods. In the present scenario, appreciated efforts have been made to explore effective dual antiviral drugs and long-acting 
(LA) drugs and vaccines to eradicate HIV infection without adverse effects. Recently, dual therapy, ie, an optimized cocktail of two ARV drugs, has been explored to manage HIV infection. A comprehensive review on the effects, advantages, and disadvantages of the dual therapy is described by Kelly et al. ${ }^{5}$ The authors claimed that the dual therapy containing tenofovir (Tef) exhibited more therapeutic advantages than triple therapy. However, the selection of appropriate drug according to the patient condition is very crucial because this therapy may reduce virologic efficacy in HIV-infected patient while lowering CD4 counts per high pre-ART HIV-1 RNA level. This report proposed that new nanoformulations (NFs) of LA cabotegravir ( $\mathrm{Ctg}$ ) and rilpivirine (Rpv) may have bright future prospects for HIV therapeutics. Such a viable dual therapy is useful to manage ART options and performance, which lowers the costs and the globally unmet needs of pill-fatigued and adherence-challenged individuals. ${ }^{5}$

LA drugs, as HIV therapeutic agents, are in practice for HIV prevention due to their effective longer therapeutic effects at a very low dose in comparison to daily consumption of ART. The strengths, weaknesses, opportunities, and challenges of LA-injectable therapies for HIV have been described excellently by Owen and Rannard. ${ }^{6}$ The pharmacology and formulation of LA Ctg were described in a comprehensive review presented by Trezza et al. ${ }^{7}$ The authors concluded that the pharmacologic profile of Ctg has great potential for the treatment and prevention of HIV-1 infection. This drug exhibited half-life of 40 days and showed therapeutic action at a low dose. Thus, monthly and bimonthly oral administration in the form of tablet would be enough to control HIV infection. ${ }^{7}$ The multi-component pharmacokinetic evaluation of LA Rpv, $300 \mathrm{mg}, 600 \mathrm{mg}$, or 1,200 mg (for 84 days), in $66 \mathrm{HIV}$-negative volunteers for preexposure prophylaxis was performed by Jackson et al. ${ }^{8}$ The authors assessed ex vivo antiviral activity of cervicovaginal lavage and claimed that each Rpv dose exhibited effective therapeutic effect, lasting till 84 days, upon exposure to plasma and genital tract. This study is useful to decide the appropriate dose for HIV therapeutics. ${ }^{8}$ In another study, the safety, tolerance, and pharmacokinetics of LA Rpv (TMC278, $300 \mathrm{mg} / \mathrm{mL}$ ) formulation on administration, single and multiple intramuscular injection, in healthy volunteers were assessed by Verloes et al. ${ }^{9}$ The outcomes of this study suggested that a clinically relevant plasma concentration of Rpv could be optimized via tuning LA formulation. ${ }^{9}$ Very recently, Margolis et a ${ }^{10}$ presented a multicenter study based on long-acting ARV treatment enabling trial, for Phase IIb, using HIV-infected adults ( $>18$ years old). The results suggested that $\mathrm{Ctg}$ plus dual nucleoside reverse transcriptase inhibitors (NRTIs) therapy exhibited antiviral activity and Ctg (30 mg, once a day) plus Rpv (25 mg, once a day) also exhibited 96 weeks longer antiviral activity similar to efavirenz plus NRTIs. The authors claimed that the injectable formulation of Rpv plus Ctg can be used as a two-drug regimen for HIV infection therapeutics. ${ }^{10}$

Besides significant advancements in ARV drug development for HIV therapeutics, very few efforts were made to develop an effective anti-HIV vaccine. Gautam et al ${ }^{11}$ demonstrated the long-term efficacy and protection evaluation of passively transferred four anti-HIV-1-neutralizing monoclonal antibodies (VRC01, VRC01-LS, 3BNC117, and 10-1074), with a single high dose $(20 \mathrm{mg} / \mathrm{kg})$, in an HIV-infected monkey model. The authors claimed that a specific single neutralizing antibody demonstrated virus reduction up to 23 days. The results of this study suggested that all four antibodies showed plasma-neutralizing efficacy, which is dependent on antibody potency and half-life. Based on the outcomes, a specific anti-HIV antibody could be selected and injected to control high-risk transmission of HIV infection. ${ }^{11}$ To avoid the adverse effects of multitherapeutic agents, efforts are being made to design novel therapeutic agents that could work singly for activation and eradication of HIV infection. Guided genes, ie, CRISPR-Cas9/gRNA system, are recently being investigated to eradicate HIV in latently infected HIV reservoirs. ${ }^{3,12,13}$ Unfortunately, these studies were performed either in vitro or in periphery. HIVinfected and latently infected HIV brain reservoirs could not be targeted due to negligible transmigration of therapeutics against HIV across the BBB. ${ }^{2}$ Therefore, efforts should be made to explore novel analytical science and nanotechnology for developing strategies to deliver therapeutics across the $\mathrm{BBB}$ to prevent neuroacquired immunodeficiency syndrome (neuroAIDS) in the brain. ${ }^{14}$

The major challenge to cure neuroHIV is the inability of effective anti-HIV therapeutic agents to cross the complex integrity of the BBB. This makes HIV, ie, neuroHIV, incurable in the brain. Specific receptor binding, ${ }^{15,16}$ focused ultrasound, ${ }^{17}$ microbubble-assisted focused ultrasound, ${ }^{18}$ and magnetic field ${ }^{19}$-based approaches have been demonstrated to open the BBB for delivery of therapeutic agent. Receptorfunctionalized therapeutic cargos are bigger in size and thus affect efficacy, while an externally stimulated approach results in transient BBB opening, which may also allow the delivery of unwanted agents to the brain. ${ }^{20}$ Such agents, in the brain, may cause additional neuroinflammation and associated dementia. Recently, a noninvasive magnetically guided approach has been demonstrated by Kaushik et al. ${ }^{20}$ In this approach, the authors injected a biocompatible dose of magnetoelectric 
nanoparticles (MENPs; $10 \mathrm{mg} / \mathrm{kg}$ ), potential drug nanocarriers (NCs), into the mice brain via tail under a static magnetic field (0 T) for 3 hours to achieve CNS delivery. The transmission electron microscopy study conducted on the brain tissue of mice showed uniform distribution of MENPs in all cell types without agglomeration (Figure 1). Hematoxylin and eosin staining, blood toxicity assessment, and neurobehavior evaluation studies confirmed that the adopted methods using MENP are safe for living beings and also did not affect motor coordination function of mice..$^{20}$

\section{A}

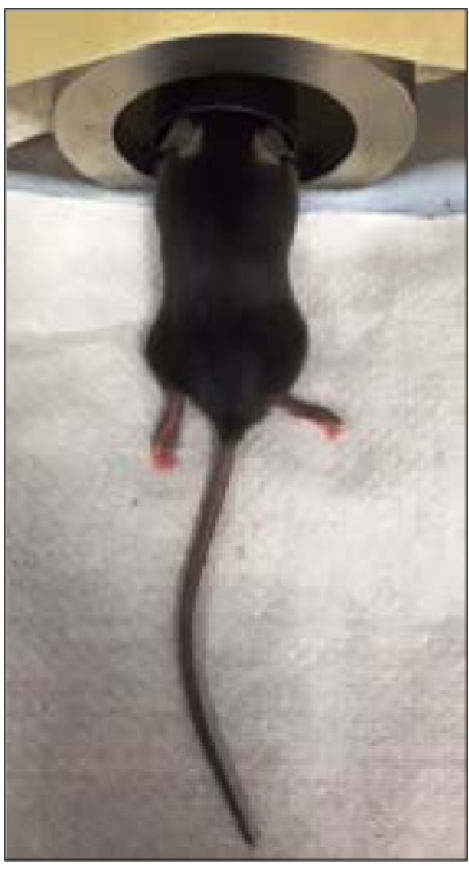

B

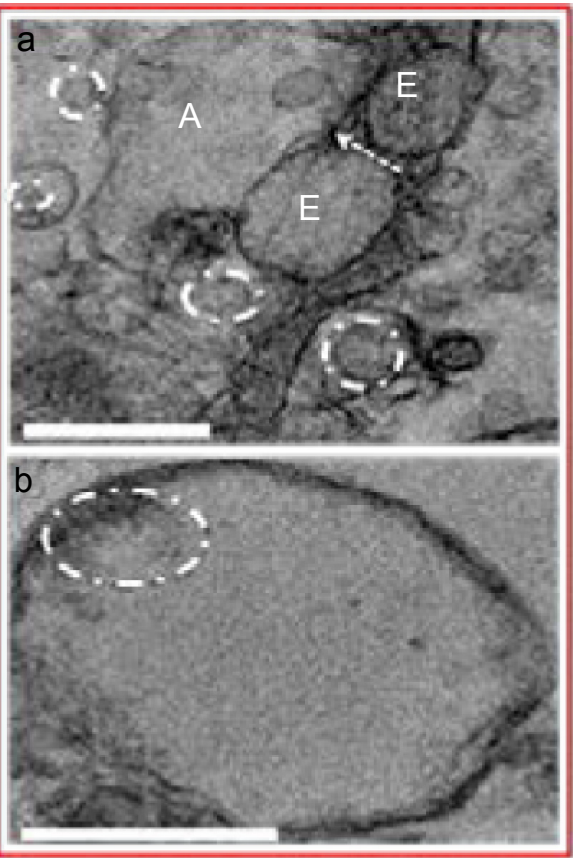

C
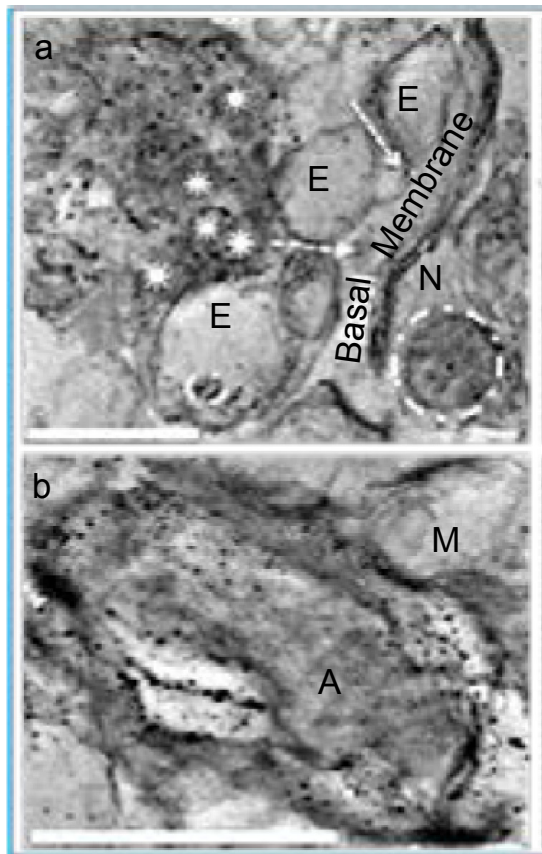
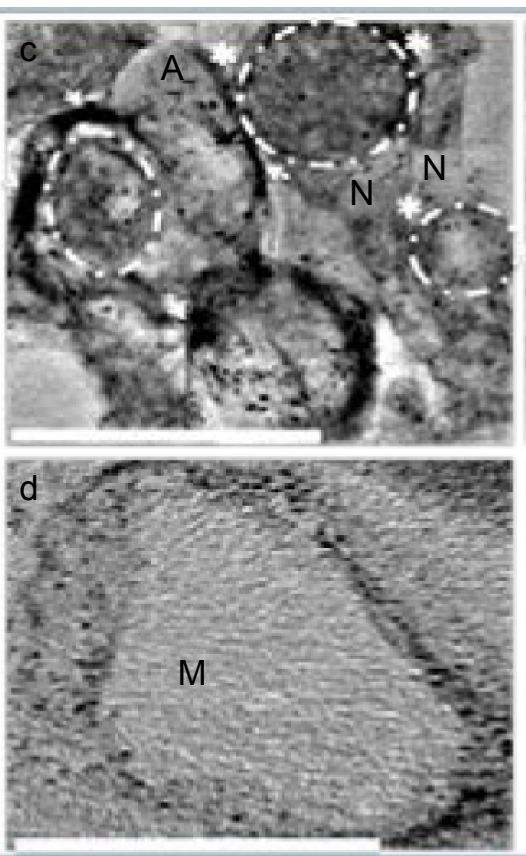
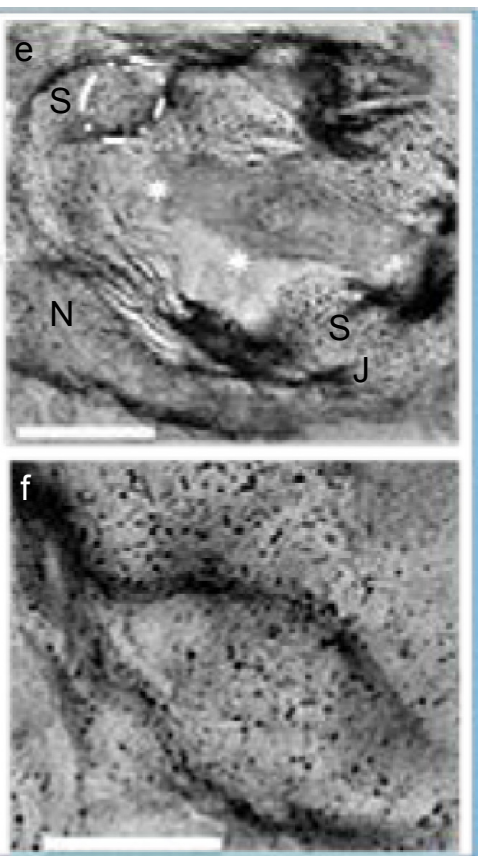

Figure I Illustration of MENP delivery to the brain under the influence of static magnetic field (A). Ex-vivo TEM image of brain tissue of control (B) and MENP-injected (C) mice.

Notes: MENPs are capable of navigating across the BBB (Ba vs $\mathbf{C a}$ ); direction of movement across tight junctions of layers of $E$ is indicated by arrows. MENPs are able to reach target sites, including $\mathrm{N}, \mathrm{A}$, and $\mathrm{M}$, and are also observed in S, E, and blood cells (Most MENPs are uniformly distributed in the brain tissue/cells and are able to reach nucleus (dotted circles), but some agglomeration of MENPs in cell membranes and their entrapment in endosomes are also observed (solid arrow heads). *Represents synapses $(\mathbf{C} c)$, J represents neuromuscular junction between $\mathbf{S}$ and the axon terminal $(\mathbf{C e})$. Scale bars: I $\mu \mathrm{m}(\mathbf{B a}$; $\mathbf{C a}, \mathrm{b}$, and e) and $0.5 \mu \mathrm{m}(\mathbf{B b}$; $\mathbf{C} c$, d, and f). Republished from Kaushik A, Jayant RD, Nikkhah-Moshaie R, et al. Magnetically guided central nervous system delivery and toxicity evaluation of magneto-electric nanocarriers. Sci Rep. 2016;6:25309.20

Abbreviations: MENP, magnetoelectric nanoparticle; TEM, transmission electron microscopy; BBB, blood-brain barrier; E, endothelial cells; N, neurons; A, astrocytes; M, microglia; S, smooth muscle cells; sc, Schwann cells; J, neuromuscular junction. 
The NCs of metal, metal oxides, gels, biopolymer, composites, and core-shell nanostructures are being designed and explored for binding of anti-HIV drugs and release of stimuli responses. ${ }^{2,20,21}$ The successful release of binded drugs is demonstrated in either a sustained or externally stimulated on-demand controlled manner. At present, developed NFs, ie, optimized combination of NCs and specific drug, were tested using an in vitro model that exhibited a high therapeutic effect and less adverse effect. ${ }^{1,21,22}$ Carson et al $^{23}$ demonstrated a tunable sustained release of multiclass water-soluble (hydrophilic) anti-HIV drugs, ie, Tef, using polyester-blended electrospun fibers as a potential drug NC. ${ }^{23}$ The authors claimed that binding and release of anti-HIV drug was dependent on the affinity level between Tef and the functional groups available on the biocompatible fiber. This developed NF is able to carry high drug loading, $\sim 40 \mathrm{wt} \%$, and demonstrated sustained release from 24 hours to 30 days. ${ }^{23}$ However, this in vitro model needs to be confirmed using an in vivo model to evaluate the ability to cross the BBB. Destache et $\mathrm{al}^{24}$ developed an NF containing Tef to prevent HIV-1 vaginal transmission in a humanized mouse model. The authors formulated Tef disoproxil fumarate at the nanoscale using a thermosensitive gel of poly(lactic-co-glycolic acid) prepared using oil-in-water emulsification. The efficacy of this formulation was tested in humanized bone marrow-liver-thymus mice. The results of this study suggested that Tef disoproxil fumarate-NF acted as an LA drug via sustained release of Tef to show coitus-independent HIV-1 vaginal protection modality. ${ }^{24}$ Freeling et a ${ }^{25}$ developed an NF containing three LA anti-HIV drugs (lopinavir, ritonavir, and Tef) loaded in a lipid nanoparticle for longer exposure in primate plasma cells within lymph nodes (persistent HIV reservoirs) and blood to cure HIV infection. This developed NF showed 50-fold higher intracellular drug concentration in lymph in comparison to free drug administration. After single administration to plasma, the authors demonstrated that the intracellular drug level was enhanced until 7 days. Thus, the presented NF can be used as oral therapy for HIV at the periphery level. ${ }^{25}$

Roy et $\mathrm{a}^{26}$ developed an NF (140 nm) to eradicate HIV infection in the gut-associated lymphoid tissue (GALT), a reservoir of early HIV infection and host-pathogen interaction. The complex physiology of GALT resisted delivery of conventional anti-HIV drugs. Thus, developing NF specific to GALT is of high therapeutic importance. The authors explored paracellular transport property of M-cells for targeted delivery of pluronic NCs containing anti-HIV drug (efavirenz) functionalized with M-cell-specific antibody of the GALT. The sustained release of anti-HIV drug significantly reduced the HIV infection level in the GALT, compared to the free drug. ${ }^{26}$ Recently, nanomedicine technology has been explored to improve the efficacy of LA Rpv via developing an NF for pericoital and coitus-independent HIV infection therapeutics, demonstrated by Kovarova et al. ${ }^{27}$ In this research, the authors binded Rpv with poly(lactic-coglycolic acid) nanoparticles and encapsulated them with a thermosensitive gel for delivery purpose. This NF, considered as solid nanosuspension of LA Rpv, was administrated intramuscularly in the bone marrow-liver-thymus mice. A single dose of this NF in mice, after a week, showed significant protection against high dose of vaginal challenge with HIV-1. The authors claimed that sometime in the presence of the antiviral drug, HIV infection may show systematic replication even on sustained release of ARV. Thus, implication of LA Rpv for HIV therapeutics is crucial and can be achieved by infecting such developed LA Rpv-based NF. ${ }^{27}$

To manage HIV-1 infection in the brain, significant efforts must be made to deliver this NF to the CNS. Numerous reports confirm that navigation across the BBB depends on the properties of NCs and NFs. ${ }^{1,2}$ Thus, exploring the properties of NCs and NFs independently is crucial to design an effective therapeutic cargo to cure HIV completely in the brain. However, the BBB always prevents the migration of NCs to the brain. Surface science has explored making the surface of NCs hydrophobic and generating functionality on NCs to bind with the BBB-related specific biomolecules, such as antibody, protein, and enzyme, to achieve CNS delivery. Externally controlled navigation strategy based on magnetic field and ultrasound strategy has recently been demonstrated for transmigration of NCs across the BBB. ${ }^{1,2}$

Recently, magnetic nanoparticles (MNPs) have been proposed as potential NCs to prepare effective NFs for the eradication of HIV in the brain and periphery. ${ }^{4,21}$ Jayant et $\mathrm{al}^{4}$ developed layer-by-layer technology-based novel NFs consisting of tef, vorinostat (a latency-breaking agent), and $\mathrm{Fe}_{3} \mathrm{O}_{4}(10 \pm 3 \mathrm{~nm})$ as NCs for the treatment of neuroAIDS. The authors demonstrated magnetically guided delivery of this NF across the BBB and sustained release of both agents to activate and then eradicate HIV infection. The outcomes of this study suggested that loading and release of drug are dependent on layer-by-layer formulation later. This would help to monitor and control the pharmacokinetics. This NF showed sustained release of therapeutic agent over a period of 5 days after HIV infection in primary human astrocytes, with good cell viability (90\%). ${ }^{4}$ Raymond et $\mathrm{a}^{28}$ demonstrated the magnetically guided delivery of Nef peptides across the BBB using MNPs as NCs to induce microglial cytokine/chemokine secretion. The exNef is a novel mediator of HIV-induced 
neuropathogenesis and could be modulated in the presence of Nef peptides. The successful delivery functionality of Nef peptides in the CNS may be useful to develop novel personalized nanomedicine for HIV therapeutics. ${ }^{28}$ Externally controlled on-demand controlled release of an NRTI, 3'-azido-3'-deoxythymidine-5' -triphosphate (AZTTP), an anti-HIV drug, was demonstrated using MENPs as a potential nanodrug carrier. An NF, consisting of MENPs and AZTTP, was formulated and magnetically delivered across the BBB. ${ }^{29}$ An external alternating current (AC) magnetic field through an electromagnetic coil was applied to release AZTTP from NF. In this mechanism, MENPs showed polarization on AC magnetic field stimulation, which changed the electrostatic bond between MENPs and the bound drug. This phenomenon occurred rapidly and repeatedly, resulting in release of the drug from the surface of MENPs. MENP-based NFs were magnetically guided across the BBB, and $100 \%$ drug release was achieved via tuning the frequency and applying AC magnetic field. This NF significantly eradicated HIV-1 infection level across the BBB and has potential to be tested using animal models. ${ }^{21,29}$ It is well known that antiviral drug in CNS induces neuroinflammatory resulted in neurotoxicity. Recently, magnetically guided delivery of an anti-inflammatory agent, namely, tissue inhibitor of metalloproteinase-1 (TIMP1), across the BBB is demonstrated to reduce HIV infection using MNPs as NCs and SK-N-MC neuroblastoma cells as in vitro model. ${ }^{30}$ This report claimed that TIMP1 reduced neuronal toxicity and significant recovery of spinal density and thus confirmed neuroprotective effects of TIMP1 in the CNS. ${ }^{30}$ The above discussed in vitro model showed impressive reduction in the HIV infection level across the BBB; therefore, efforts must be made to demonstrate these models in vivo to develop personalized nanomedicine to cure neuroHIV.

Besides successful in vitro demonstration, an NF, prepared using biocompatible nanogels binded with NRTIs, was developed by Senanayake et al, ${ }^{31}$ and they claimed this NF as a novel antiviral agent of high efficacy to combat against HIV infection. This group developed a cationic nanogel, ie,

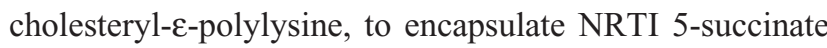
derivatives to evaluate anti-HIV activity using in vitro microphage model in the CNS as well. This NF demonstrated tenfold suppression in reverse transcriptase activity (EC90) in HIV-infected macrophage in comparison to single/double/ triple drug cocktails recommended for clinical trials. ${ }^{31}$ The sustained release of NRTIs for long time, enhanced efficacy, and biocompatible nature proposed this NF as a promising candidate to be tested in humanized mouse (hu-PBL) HIV model for the development of personalized nanomedicine. Recent success in the CNS delivery of ARV, ie, zidovudine (AZT), through the nasal route using chitosan microparticles (CP), prepared by spray drying techniques, as a carrier to cure brain HIV sanctuaries has been reported by Dalpiaz et al..$^{32}$ The authors produced a prodrug via conjugating AZT with ursodeoxycholic acid (UCDA-AZT) to overcome the issue of active efflux transporter systems. The UCDA-AZT $(5 \mu \mathrm{M}$ or $10 \mu \mathrm{M})$ system demonstrated 20 times higher efficacy than AZT to cure HIV in the CNS and in macrophages..$^{32}$ To demonstrate delivery to rat brain, the authors administered this prodrugencapsulated CP through the nasal route, aiming to achieve high CNS uptake. The results suggested that utilization of $\mathrm{CP}$ increased the dissolution rate of UDCA-AZT and reduced water uptake, resulting in high levels in cerebrospinal fluid in rats, which is required for better efficacy in the CNS. ${ }^{32}$

Currently, scientists are exploring novel multi-functional NFs to block unwanted disorders in HIV-infected patients using substance of abuse..$^{33}$ Sagar et $a 1^{33}$ developed a highly selective magnetic NF for the delivery of a therapeutic agent to block the effects induced by morphine. A morphine antagonist, ie, D-Pen-Cys-Tyr-DTrp-Orn-Thr-Pen-Thr-NH2 (CTOP), was bound with MNP and magnetically delivered across the BBB. Over time, release of CTOP showed significant efficacy via protecting modulation of neuronal dendrite and spinal density. This NF was tested in HIV-infected cell lines, and outcomes were similar to those obtained in normal cell lines. In future, efforts must be made toward the encapsulation of NCs, anti-HIV drugs, and antagonists against substances of abuse to develop an effective NF. Such developed NFs would be capable of eradicating HIV infection and to block the effects induced by substances of abuse in HIV patients. The state-of-the-art and possible future strategy to eradicate and manage HIV is shown in Figure 2.

Nanoengineered NCs, highly sensitive technologies, and smart assays are being explored to develop an ideal nanomedicine via improving properties of NCs, pharmacokinetics, and acceptable neurobehavioral alteration. ${ }^{34}$ During virus progression, the dysfunction of related biomarkers and genes has been quantified using sensitive analytical assays, namely, polymerase chain reaction, and enzyme-linked immunosorbent assay. Ultrasensitive histopathology, immunohistopathology, and optical fluorescence imaging have been adopted recently to explore the biodistribution, pathogenesis, and toxicity of both NCs and NFs. Recently, transmission electron microscopy (Figure 1) with illuminating features was introduced to study the distribution of NCs in various organs to explain particle-to-particle interaction and elemental 


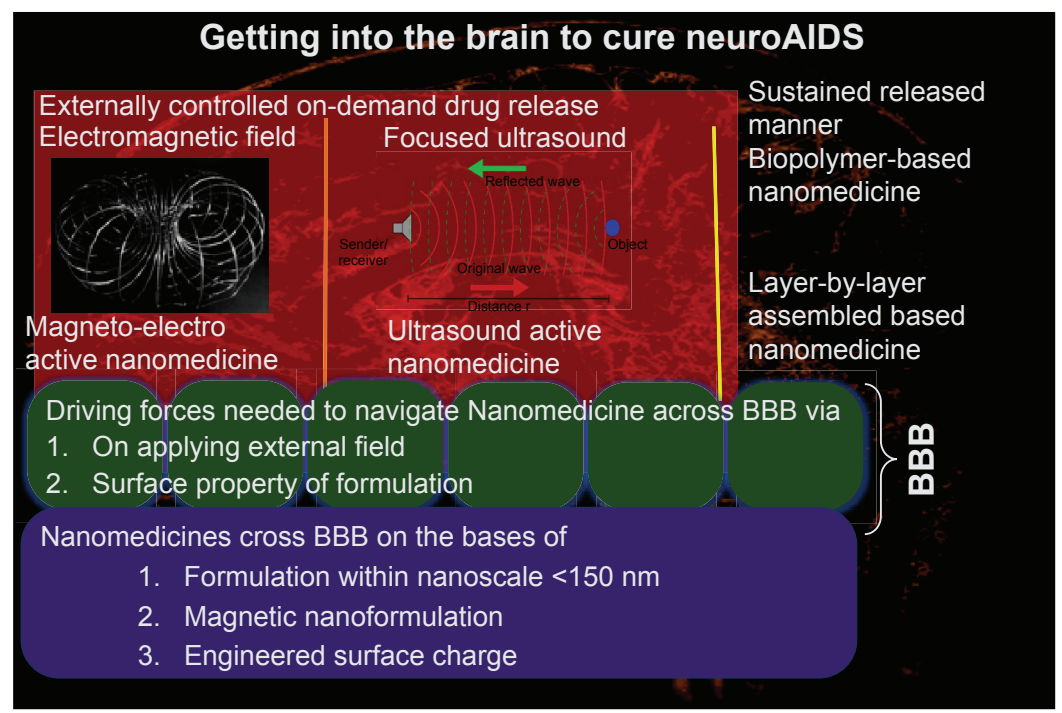

Figure 2 State-of-the-art personalized nanomedicine to cure HIV and neuroAIDS

Abbreviations: HIV, human immunodeficiency virus; neuroAIDS, neuroacquired immunodeficiency syndrome; BBB, blood-brain barrier.

composition of NCs over time after injection. ${ }^{20}$ Biodistribution and therapeutic effects of contrast agents containing NCs and NFs in the periphery and brain were studied using magnetic resonance imaging. The homeostatic effect of NCs in the living system was also studied using magnetic resonance imaging via real-time monitoring of contrast exposure.

It has been reported that HIV infection is serious, but it turns worst in combination with drugs of abuse. For example, HIV patients due to life style and ingestion of drug of abuse were found to be psychologically stressed which resulted in delayed response to therapeutics. ${ }^{35}$ Thus, stress monitoring in HIV patients is crucial to decide therapeutics. Stress management of HIV patients has been declared necessary to obtain information for clinicians to avoid delay in therapeutics. ${ }^{35}$ The addition of such analytical devices is new to HIV therapy, but clinicians expect bright future prospects to assess social assessment impact of patients. ${ }^{36}$ The introduction of analytical sensing systems for HIV incidence will be of great significance. ${ }^{36,37}$ Enzyme-linked immunosorbent assay and real-time/quantitative polymerase chain reaction have demonstrated estimation of potential HIV biomarkers such as HIV-1 RNA viral load and CD4 cell counts. These methods have also quantified other HIV-infection-related biomarkers such as serum concentration of neopterin, beta- 2 microglobulin (IgA), interleukin-2 receptor, CD8 cell counts, anti-HIV antibodies, p24 antigen, hemoglobin level, platelet concentration, and erythrocyte sedimentation rate. Both these methods are very well established, but the need for high operation expertise and longer analysis time has raised the demand for rapid diagnostic tools to detect/monitor HIV infection in the physiological range. The available detection tools for detection of HIV biomarkers with performance parameters are summarized in Table 1.

Recently, electrochemical biosensing system has been developed to detect physiological stress markers in HIV patients. The electrochemical biosensors recently emerged as a potential analytical tool to detect target biomarkers at the picomolar level..$^{38-41}$ Shafiee et al have described the available potential techniques to detect HIV biomarkers to estimate HIV infection level. This group also proposed that the development of electrochemical system with features of affordability, sensitivity, specificity, user friendliness, rapidity, robustness, equipment-freeness, and desirability can be promoted at pointof-care application to detect HIV infection for personalized health care.$^{36}$ The performance of available detection tools to detect and monitor HIV infection is summarized in Table 2.

As per state of the art and according to ASSURED, a biosensor fabricated using a nanoplasmonic resonance detected $\sim 100$ copies/mL of HIV-1 subtype D using a very small volume $(100 \mu \mathrm{L})$ of whole blood and plasma. ${ }^{42}$ A label-free optical biosensor developed using a nanophotonic crystal was used to detect $10^{4}-10^{6}$ copies/mL of HIV-1 in blood plasma. ${ }^{43}$ Such nanoenabled biosensors were operated simply with the salient feature of integration with nanosensing components such as nanoelectronics, miniaturized transduction techniques, and microelectromechanical systems for detection of potential HIV-infection biomarkers $\left(\mathrm{CD}^{+} \mathrm{T}\right.$ lymphocytes and viral load) in the real sample, even at the point-of-care application. ${ }^{44-46}$ Using lab-on-chip-based approach, the capturing and quantification of $\mathrm{CD}^{+}$lymphocytes has been demonstrated by Watkins et al. ${ }^{47}$ The authors reported that lab-on-chip functionalized by a specific antibody detected 
Table I Available analytical tools for detection of HIV infection

\begin{tabular}{|c|c|c|c|}
\hline Techniques & $\begin{array}{l}C D^{+} T \text { lymphocytes } \\
\text { counting }\end{array}$ & $\begin{array}{l}\text { Viral load } \\
\text { estimation }\end{array}$ & Remarks \\
\hline Flow cytometry & $\begin{array}{l}\text { Conventionally well established } \\
\text { for both }\end{array}$ & & $\begin{array}{l}\text { - Assay time: } 8-40 \text { minutes } \\
\text { - Sample volume: } 10 \mu \mathrm{L} \text { of blood } \\
\text { - Laboratory set-up } \\
\text { - Not suitable for POC }\end{array}$ \\
\hline Image processing & $\begin{array}{l}\text { Bright field or fluorescent } \\
\text { image of } C D^{+} T \text { lymphocytes }\end{array}$ & & $\begin{array}{l}\text { - Assay time: } 20-30 \text { minutes } \\
\text { - Sample volume: } 30 \mathrm{~mL} \text { of whole blood } \\
\text { - Power: AC battery } \\
\text { - Only qualitative } \\
\text { - Not suitable for POC }\end{array}$ \\
\hline ELISA & Centrifuge-based approach & Nanoenabled ELISA & $\begin{array}{l}\text { - Assay time: } 40 \text { minutes for } \mathrm{CD}^{+} \text {and } 48 \text { hours for viral load } \\
\text { - Sample volume: } 30 \mu \mathrm{L} \text { of blood and I } \mathrm{mL} \text { of plasma } \\
\text { - Power: DC battery }\end{array}$ \\
\hline RT-PCR & & $\begin{array}{l}\text { DNA based } \\
\text { virus capture based }\end{array}$ & $\begin{array}{l}\text { - Assay time: } 35-90 \text { minutes } \\
\text { - Sample volume: } 150 \mu \mathrm{L} \text { of blood, } 75 \mu \mathrm{L} \text { of fingerstick } \\
\text { blood, and I } \mathrm{mL} \text { of plasma } \\
\text { - Power: AC/DC battery }\end{array}$ \\
\hline Electrical sensing & $\begin{array}{l}\text { Best suitable for HIV detection } \\
\text { and monitoring }\end{array}$ & & $\begin{array}{l}\text { - Sensing time: } 15-30 \text { minutes } \\
\text { - Sample volume: } 10 \mu \mathrm{L} \\
\text { - Power: AC battery/solar } \\
\text { - Sensitive and selective } \\
\text { - Suitable for detection and monitoring } \\
\text { - Suitable for POC application }\end{array}$ \\
\hline
\end{tabular}

Abbreviations: HIV, human immunodeficiency virus; POC, point of care; AC, alternating current; ELISA, enzyme-linked immunosorbent assay; DC, direct current; RT-PCR, reverse transcription polymerase chain reaction.

viral lysate ranging from 100 cells $/ \mathrm{mL}$ to 700 cells $/ \mathrm{mL}$, with a detection limit of 9 cells $/ \mu \mathrm{L}$ and a detection range from 100 cells $/ \mathrm{mL}$ to 700 cells $/ \mu \mathrm{L} .{ }^{47}$ However, the detection of neuroHIV infection and also HIV incidences was not well explored by these sensing systems. Significant efforts should be made to investigate biomarkers for the detection of neuroHIV infection. The significance of neuroHIV monitoring and management using advanced nanoenabling sensing systems is described by Nair et al, ${ }^{1}$ as shown in Figure 3 . Recently, electrochemical monitoring of HIV-infection in presence

Table 2 A performance summary of available diagnostic tests/tools used to detect HIV infection

\begin{tabular}{|c|c|}
\hline Diagnostic test/tool & Detection technique and sensitivity/specificity \\
\hline $\begin{array}{l}\text { Low-cost, rapid HIV-monitoring tests: } \\
\text { - OraQuick Rapid HIV-I/2 Antibody Test } \\
\text { - Aware HIV-I/2 U (alternative to urine tests) } \\
\text { - Anti-HIV antibody and p } 24 \text { antigen (for early infant diagnosis and for HIV-I and } \\
\text { HIV-2 subtype differentiation) } \\
\text { - HIV-I/2 Ag/Ab Combo (antigen and antibody) }\end{array}$ & $\begin{array}{l}\text { - Oral fluids/blood sample }(92.7 \% / / 00 \%) \\
\text { - Blood/serum }(88.7 \% / 99.9 \%) \\
\text { - ELISAs (I2.5-200 pg/mL) } \\
\text { - Immunochromatographic }(50 \% / 86 \%)\end{array}$ \\
\hline $\begin{array}{l}\text { HIV viral load monitoring: } \\
\text { - RT assay: (ExaVir Load) } \\
\text { - NAT: i) COBAS Amplicor HIV-I Monitor vI.5; ii) COBAS AmpliPrep/COBAS } \\
\text { TaqMan v2.0; iii) Real-Time HIV-I; and iv) VERSANT HIV-I RNA I.0 Assay (kPCR) } \\
\text { - Non-NAT (HIV viral enzymes and proteins) } \\
\text { - p24 antigen technologies (NEN HIV-I p24 ELISA) } \\
\text { - } \text { p24 antigen + anti-HIV, IgM, and IgG antibodies (HIV-I/2Ag/Ab Combo) }\end{array}$ & $\begin{array}{l}\text { - RT activity in blood } 200-600,000 \text { copies } / \mathrm{mL} \\
\text { - Detect and quantify viral RNA via RT-PCR } \\
\text { - } 50-750,000 \text { copies/mL with } 99.85 \% \text { specificity } \\
\text { - N/A } \\
\text { - Detect and quantify HIV viral enzymes and proteins } \\
\text { ( } 10,000-30,000 \text { RNA copies } / \mathrm{mL}) \\
\text { - Detect blood p24 antigen }(30,000-50,000 \text { RNA copies } / \mathrm{mL})\end{array}$ \\
\hline $\begin{array}{l}\text { Microfluidics diagnostic: } \\
\text { - p24 test for EID } \\
\text { - CD4 } 4^{+} \text {cells from whole blood (Daktari Diagnostics) }\end{array}$ & $\begin{array}{l}\text { - Plasma }(95 \% / 99 \%) \\
\text { - Whole blood }(97 \% / 86 \%)\end{array}$ \\
\hline $\begin{array}{l}\text { POC: } \\
\text { - Ultrasensitive p24 Antigen Assay (NWGHF) } \\
\text { - PanNAT Diagnostic Platform } \\
\text { - Portable CD4+ T-cell count (PointCare NOW, CyFlow miniPOC) }\end{array}$ & $\begin{array}{l}\text { - } \text { Heel-stick blood }(95 \% / 99 \%) \\
\text { - } \text { Blood }(95 \% / 99 \%) \\
\text { - } \text { Blood }(90 \% / 96 \%)\end{array}$ \\
\hline
\end{tabular}

Abbreviations: HIV, human immunodeficiency virus; ELISA, enzyme-linked immunosorbent assay; RT, reverse transcription; NAT, nucleic acid-based tests; PCR, polymerase chain reaction; N/A, not applicable; EID, early infant diagnosis; POC, point of care. 


\section{NeuroAIDS monitoring and management}

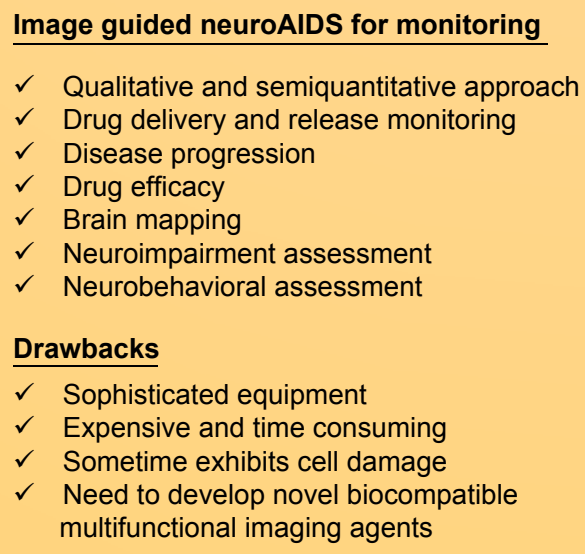

\section{Nanoenabled smart assay for neuroAIDS management}

$\checkmark$ Selective and sensitive quantitative approach

$\checkmark$ Portable and can be used at POC

$\checkmark$ ARV therapy

$\checkmark$ Viral load; $C D^{+}$counts

$\checkmark$ Drug resistance

$\checkmark$ Mother-to-child transmission

$\checkmark$ Provide bioinformatics for diagnostics

\section{Drawbacks}

$\checkmark \quad$ Need to optimize device integration with reduced form factors

Figure 3 Performance comparison of analytical tools used to monitor and detect neuroHIV/AIDS.

Note: Reprinted from Adv Drug Deliv Rev, 103/I, Nair M, Jayant RD, Kaushik A, Sagar V, Getting into the brain: potential of nanotechnology in the management of neuroAIDS, 202-217, Copyright (2016), with permission from Elsevier.'

Abbreviations: ARV, antiretroviral; neuroHIV, neurohuman immunodeficiency virus; AIDS, acquired immunodeficiency syndrome; BBB, blood-brain barrier; POC, point of care.

of cocaine and specific drugs related with HIV-infection and cocaine was demonstrated using an elctrochemical monitoring-on-chip (E-Moc) approach. Authors developed a cultureware chip and human astrocytes based in-vitro model for HIV-infection, Cocaine explore, treatment with Tef, along with rimcazole (RA, a cocaine antagonist). Electrochemical impedance spectroscopy was performed to evaluate electrophysiology of cells during infection and therapeutic. Author claimed this methodology as an effective analytical tool for HIV-infection monitoring in various conditions. ${ }^{48}$

In summary, to cure HIV and neuroHIV/AIDS, we believe that significant research must be performed to design novel biocompatible NFs with salient features of target site delivery, novel easy mechanism to release ARV drugs, and effective CNS delivery to eradicate latent HIV infection. The investigation of novel diagnostic tools for detection of infection and its integration with NF-based HIV eradication will be useful to assess NF functionality with real-time monitoring of HIV infection. These significant advancements will help to decide HIV therapeutics and management. Therefore, we can say that involvement of new analytical diagnostic tools and nanoenabling formulations will simplify both HIV diagnosis and cure to manage personalized care.

\section{Acknowledgment}

The authors acknowledge the Institute of NeuroImmune Pharmacology, Department of Immunology, Herbert Wertheim College of Medicine, Florida International University, for encouragement and support.

\section{Disclosure}

The authors report no conflicts of interest in this work.

\section{References}

1. Nair M, Jayant RD, Kaushik A, Sagar V. Getting into the brain: potential of nanotechnology in the management of neuroAIDS. Adv Drug Deliv Rev. 2016;103(1):202-217.

2. Ruiz A, Nair M, Kaushik A. Recent update in NanoCure of neuroAIDS. Sci Lett J. 2015;4:172.

3. Zhang Y, Yin C, Zhang T, et al. CRISPR/gRNA-directed synergistic activation mediator (SAM) induces specific, persistent and robust reactivation of the HIV-1 latent reservoirs. Sci Rep. 2015;5:16277.

4. Jayant RD, Atluri VS, Agudelo M, Sagar V, Kaushik A, Nair M. Sustained-release nanoART formulation for the treatment of neuroAIDS. Int J Nanomedicine. 2015;10:1077-1093.

5. Kelly SG, Nyaku AN, Taiwo BO. Two-drug treatment approaches in HIV: finally getting somewhere? Drugs. 2016;76(5):523-531.

6. Owen A, Rannard S. Strengths, weaknesses, opportunities and challenges for long acting injectable therapies: insights for applications in HIV therapy. Adv Drug Deliv Rev. Epub 2016 Feb 23.

7. Trezza C, Ford SL, Spreen W, Pan R, Piscitelli S. Formulation and pharmacology of long-acting cabotegravir. Curr Opin HIV AIDS. 2015;10(4): 239-245.

8. Jackson AG, Else LJ, Mesquita PM, et al. A compartmental pharmacokinetic evaluation of long-acting rilpivirine in HIV-negative volunteers for pre-exposure prophylaxis. Clin Pharmacol Ther. 2014;96(3): 314-323.

9. Verloes R, Deleu S, Niemeijer N, Crauwels H, Meyvisch P, Williams P. Safety, tolerability and pharmacokinetics of rilpivirine following administration of a long-acting formulation in healthy volunteers. HIV Med. 2015;16(8):477-484.

10. Margolis DA, Brinson CC, Smith GH, et al. Cabotegravir plus rilpivirine, once a day, after induction with cabotegravir plus nucleoside reverse transcriptase inhibitors in antiretroviral-naive adults with HIV-1 infection (LATTE): a randomised, phase $2 \mathrm{~b}$, dose-ranging trial. Lancet Infect Dis. 2015;15(10):1145-1155.

11. Gautam R, Nishimura Y, Pegu A, et al. A single injection of anti-HIV-1 antibodies protects against repeated SHIV challenges. Nature. 2016; 533(7601):105-109. 
12. Hu W, Kaminski R, Yang F, et al. RNA-directed gene editing specifically eradicates latent and prevents new HIV-1 infection. Proc Natl Acad Sci US A. 2014;111(31):11461-11466.

13. Kaminski R, Chen Y, Fischer T, et al. Elimination of HIV-1 genomes from human T-lymphoid cells by CRISPR/Cas9 gene editing. Sci Rep. 2016;6:22555.

14. Jayant RD, Sosa D, Kaushik A, et al. Current status of non-viral gene therapy for CNS disorders. Expert Opin Drug Deliv. Epub 2016 Jun 1.

15. Peluffo H, Unzueta U, Negro-Demontel ML, et al. BBB-targeting, protein-based nanomedicines for drug and nucleic acid delivery to the CNS. Biotechnol Adv. 2015;33(2):277-287.

16. Rao KS, Reddy MK, Horning JL, Labhasetwar V. TAT-conjugated nanoparticles for the CNS delivery of anti-HIV drugs. Biomaterials 2008;29(33):4429-4438.

17. Mead B, Mastorakos P, Suk JS, Song J, Hanes J, Price R. Localized delivery of non-viral gene-bearing nanoparticles into the rat brain following focused ultrasound-mediated BBB opening. J Ther Ultrasound. 2015;3(suppl 1):29.

18. Mayer CR, Geis NA, Katus HA, Bekeredjian R. Ultrasound targeted microbubble destruction for drug and gene delivery. Expert Opin Drug Deliv. 2008;5(10):1121-1138.

19. Tabatabaei SN, Girouard H, Carret A-S, Martel S. Remote control of the permeability of the blood-brain barrier by magnetic heating of nanoparticles: a proof of concept for brain drug delivery. J Control Release. 2015;206:49-57.

20. Kaushik A, Jayant RD, Nikkhah-Moshaie R, et al. Magnetically guided central nervous system delivery and toxicity evaluation of magnetoelectric nanocarriers. Sci Rep. 2016;6:25309.

21. Kaushik A, Jayant RD, Sagar V, Nair M. The potential of magnetoelectric nanocarriers for drug delivery. Expert Opin Drug Deliv. 2014; 11(10):1635-1646.

22. Roy U, Rodríguez J, Barber P, das Neves J, Sarmento B, Nair M. The potential of HIV-1 nanotherapeutics: from in vitro studies to clinical trials. Nanomedicine. 2015;10(24):3597-3609.

23. Carson D, Jiang Y, Woodrow KA. Tunable release of multiclass antiHIV drugs that are water-soluble and loaded at high drug content in polyester blended electrospun fibers. Pharm Res. 2016;33(1):125-136.

24. Destache CJ, Mandal S, Zhe Y, et al. Topical tenofovir disoproxil fumarate (DF) nanoparticles prevents HIV-1 vaginal transmission in the humanized mouse model. Antimicrob Agents Chemother. 2016;60(6):3633-3639.

25. Freeling JP, Koehn J, Shu C, Sun J, Ho RJ. Long-acting three-drug combination anti-HIV nanoparticles enhance drug exposure in primate plasma and cells within lymph nodes and blood. AIDS. 2014;28(17):2625-2627.

26. Roy U, Ding H, Pilakka-Kanthikeel S, et al. Preparation and characterization of anti-HIV nanodrug targeted to microfold cell of gut-associated lymphoid tissue. Int J Nanomed. 2015;10:5819-5835.

27. Kovarova M, Council OD, Date AA, et al. Nanoformulations of rilpivirine for topical pericoital and systemic coitus-independent administration efficiently prevent HIV transmission. PLoS Pathog. 2015;11(8): e1005075

28. Raymond A, Diaz P, Chevelon S, et al. Microglia-derived HIV Nef+ exosome impairment of the blood-brain barrier is treatable by nanomedicine-based delivery of Nef peptides. J Neurovirol. 2015; 22:129-139.

29. Nair M, Guduru R, Liang P, Hong J, Sagar V, Khizroev S. Externally controlled on-demand release of anti-HIV drug using magneto-electric nanoparticles as carriers. Nat Commun. 2013;4:1707.

International Journal of Nanomedicine

\section{Publish your work in this journal}

The International Journal of Nanomedicine is an international, peerreviewed journal focusing on the application of nanotechnology in diagnostics, therapeutics, and drug delivery systems throughout the biomedical field. This journal is indexed on PubMed Central, MedLine, CAS, SciSearch $®$, Current Contents $\AA /$ Clinical Medicine,
30. Atluri SV, Jayant DR, Pilakka-Kanthikeel S, et al. Development of tissue inhibitor of metalloproteinase-1 (TIMP-1) magnetic nanoformulation for regulation of synaptic plasticity in HIV-1 infection. Int $J$ Nanomedicine. In Press 2016.

31. Senanayake TH, Gorantla S, Makarov E, Lu Y, Warren G, Vinogradov SV. Nanogel-conjugated reverse transcriptase inhibitors and their combinations as novel antiviral agents with increased efficacy against HIV-1 infection. Mol Pharm. 2015;12(12):4226-4236.

32. Dalpiaz A, Fogagnolo M, Ferraro L, et al. Nasal chitosan microparticles target a zidovudine prodrug to brain HIV sanctuaries. Antiviral Res. 2015; 123:146-157.

33. Sagar V, Pilakka-Kanthikeel S, Atluri VS, et al. Therapeutical neurotargeting via magnetic nanocarrier: implications to opiate-induced neuropathogenesis and neuroAIDS. J Biomed Nanotechnol. 2015;11(10): 1722-1733.

34. Jayant R, Nair M. Role of biosensing technology for neuroAIDS management. J Biosens Bioelectron. 2016;7(1):e141.

35. Kaushik A, Yndart A, Jayant RD, et al. Electrochemical sensing method for point-of-care cortisol detection in human immunodeficiency virusinfected patients. Int J Nanomedicine. 2015;10:677-685.

36. Shafiee $\mathrm{H}$, Wang $\mathrm{S}$, Inci $\mathrm{F}$, et al. Emerging technologies for point-of-care management of HIV infection. Annu Rev Med. 2015;66:387-405.

37. Yager P, Domingo GJ, Gerdes J. Point-of-care diagnostics for global health. Annu Rev Biomed Eng. 2008;10:107-144.

38. Kaushik A, Tiwari S, Jayant RD, Marty A, Nair M. Towards detection and diagnosis of Ebola virus disease at point-of-care. Biosens Bioelectron. 2016;75:254-272.

39. Kaushik A, Vasudev A, Arya SK, Pasha SK, Bhansali S. Recent advances in cortisol sensing technologies for point-of-care application. Biosens Bioelectron. 2014;53:499-512.

40. Kaushik A, Jayant RD, Tiwari S, Vashist A, Nair M. Nano-biosensors to detect beta-amyloid for Alzheimer's disease management. Biosens Bioelectron. 2016;80:273-287.

41. Cruz AFD, Norena N, Kaushik A, Bhansali S. A low-cost miniaturized potentiostat for point-of-care diagnosis. Biosens Bioelectron. 2014;62: 249-254.

42. Inci $\mathrm{F}$, Tokel $\mathrm{O}$, Wang $\mathrm{S}$, et al. Nanoplasmonic quantitative detection of intact viruses from unprocessed whole blood. ACS Nano. 2013;7(6): 4733-4745.

43. Shafiee H, Lidstone EA, Jahangir M, et al. Nanostructured optical photonic crystal biosensor for HIV viral load measurement. Sci Rep. 2014;4:4116.

44. Cheng X, Liu Y-S, Irimia D, et al. Cell detection and counting through cell lysate impedance spectroscopy in microfluidic devices. Lab Chip. 2007;7(6):746-755.

45. Holmes D, Morgan H. Single cell impedance cytometry for identification and counting of CD4 T-cells in human blood using impedance labels. Anal Chem. 2010;82(4):1455-1461.

46. Shafiee H, Jahangir M, Inci F, et al. Acute on-chip HIV detection through label-free electrical sensing of viral nano-lysate. Small. 2013;9(15): 2553-2563.

47. Watkins NN, Sridhar S, Cheng X, et al. A microfabricated electrical differential counter for the selective enumeration of CD4+ T lymphocytes. Lab Chip. 2011;11(8):1437-1447.

48. Kaushik A, Vabbina PK, Atluri V, et al. Electrochemical monitoring-onchip (E-MoC) of HIV-infection in presence of cocaine and therapeutics. Biosens Bioelectron. 2016;86:426-431.

\section{Dovepress}

Journal Citation Reports/Science Edition, EMBase, Scopus and the Elsevier Bibliographic databases. The manuscript management system is completely online and includes a very quick and fair peer-review system, which is all easy to use. Visit http://www.dovepress.com/ testimonials.php to read real quotes from published authors. 